\title{
AUTOMATIC EXTRACTION OF A NAVIGATION GRAPH INTENDED FOR INDOORGML FROM AN INDOOR POINT CLOUD
}

\author{
P. Flikweert ${ }^{1,2 *}$, R. Peters ${ }^{3}$, L. Díaz-Vilariño ${ }^{4,5}$, R. Voûte ${ }^{2,5}$, B. Staats ${ }^{2}$ \\ ${ }^{1}$ Master of Science Geomatics, Faculty of Architecture and the Built Environment, TU Delft, The Netherlands - puck.flikweert@tudelft.nl \\ ${ }^{2}$ CGI Nederland BV, The Netherlands (puck.flikweert, robert.voute, bart.staats)@cgi.com \\ ${ }^{3}$ Department of Urbanism, Faculty of Architecture and the Built Environment, TU Delft, The Netherlands - r.y.peters@tudelft.nl \\ ${ }^{4}$ Applied Geotechnologies Group, Dept. of Natural Resources and Environmental Engineering, University of Vigo, Spain - lucia@uvigo.es \\ ${ }^{5}$ Department of OTB, Faculty of Architecture and the Built Environment, TU Delft, The Netherlands - (1.diaz-vilarino, r.voute)@tudelft.nl
}

\section{Commission IV, WG IV/5}

KEY WORDS: indoor navigation, IndoorGML, navigation graph, point cloud, voxels, indoor, laser scanning, 3D Medial Axis Transform, automatic door detection

\begin{abstract}
:
Indoor environments tend to be more complex and more populated when buildings are accessible to the public. The need for knowing where people are, how they can get somewhere or how to reach them in these buildings is thus equally increasing. In this research point clouds are used, obtained by dynamic laser scanning of a building, since we cannot rely on architectural drawings for maps and paths, which can be outdated. The presented method focuses on the creation of an indoor navigation graph, based on IndoorGML structure, in a fast and automated way, while retaining the type of walkable surface. In this paper the focus has been on door detection, because doors are essential elements in an indoor environment, seeing that they connect spaces and are a logical step in a route. This paper describes a way to detect doors using 3D Medial Axis Transform (MAT) combined with the intelligence stored in the path of a mobile laser scanner, showing good first results. Additionally different spaces (e.g. rooms and corridors) in the building are identified and slopes and stairs in walkable spaces are detected. This results in a navigation graph which can be stored in an IndoorGML structure.
\end{abstract}

\section{INTRODUCTION}

In the past years there has been an increasing need for navigation assistance in indoor environments, because of the growing complexity and size of buildings. It can especially be helpful to wheelchair users, who are restricted in their routes, or for blind or partially sighted people, who are not always able to follow route signs (Mirza et al., 2012). Moreover Boguslawski et al. (2016) stress the importance of up-to-date indoor models of complex buildings for disaster scenarios and emergency response.

However, existing building models or floor plans are often outdated and can contain wrong information, because the situation "as-built" can differ from the way that it was planned (Bosché et al., 2014; Volk et al., 2014; Hong et al., 2015; Alattas et al., 2017; Staats et al., 2018). Besides manually updating floor plans or building models, a less labour-intensive way to obtain up-todate information on an indoor environment is to make use of point clouds. In order to obtain them one can use static or dynamic laser scanners, the advantage of the first being higher accuracy of the points. However, because the laser scanner is only set up at limited locations in the environment, the resulting point cloud can miss certain parts of the environment that are occluded by furniture and other objects. Dynamic laser scanning provides better opportunities for a faster and more complete coverage of the environment, because one can also walk behind objects that are occluding part of the environment, such as furniture. Point cloud processing algorithms are needed to obtain useful information from the millions of points, such as the shape and location of elements in the indoor environment. This information in turn can be used for navigation purposes.

\footnotetext{
${ }^{*}$ Corresponding author
}

In this paper a process is described to go from an indoor point cloud to a navigation graph based on a structure defined in the IndoorGML standard. In this process walkable voxels (Staats et al., 2017, 2018), obtained through a region growing method, are generalised into different rooms by doorway detection, and from this a graph is constructed between indoor spaces. A novel way of door detection in point clouds is described, making use of the trajectory of the laser scanner and the 3D Medial Axis Transform (MAT) (Peters, 2018) of the point cloud. Related work on the topic of this paper is discussed in Section 2. Background information on theories directly applied in this research, such as IndoorGML, voxels, walkable space detection and the 3D MAT of point clouds is described in Section 3, after which the door detection method and network generation are explained in Section 4. Results and a discussion of them are given in Section 5, and finally conclusions and future work are discussed in Section 6.

\section{RELATED WORK}

\subsection{Indoor navigation networks}

Essential requirements for indoor navigation are: a way to localise the person to be navigated (indoor positioning), a map or 3D model of the environment, a network graph representing navigable paths, a path-finding algorithm and a human interpretation of the path (wayfinding) (Nakagawa and Nozaki, 2018). This paper focuses on the third: creating a navigation graph for an indoor environment, in which nodes represent locations, and edges the connectivity between these locations. This graph can be used to calculate optimal routes between nodes, in order to navigate people through buildings. 
Sithole (2018) and Nakagawa and Nozaki (2018) describe three main types of navigation networks found in related work: 1) graphs that link separate indoor spaces (e.g. rooms and corridors), 2) grid structured graphs that are based on small-scale cells or voxels (tessellation), and 3) potential field models, which are continuous and have repelling (obstacles, walls) and attracting (the end goal) forces. The latter is most often used in the navigation of autonomous robots and drones (Koren and Borenstein, 1991; Ge and Cui, 2002), and is computationally very heavy for route calculation. Grid structured graphs can be based on 2D cells (pixels), as done by Nakagawa and Nozaki (2018), limited to one floor, or voxels (3D volumetric pixels), as done by Fichtner et al. (2018). The last authors create an octree structure in the empty space of a point cloud, and construct a connectivity graph in neighbouring empty octree leaves. The smaller the leaves, the more accurate information can be represented. However, depending on the chosen cell size, resulting networks are often dense. Because of this route calculation time in large buildings can quickly increase. Moreover these networks do not contain any knowledge on the presence of stairs, doors and other important navigation concepts, and they are often unnecessarily small-scaled for human navigation purposes. This is why this research focuses on the first type of network: graphs linking separate indoor spaces.

Duality is a main theme in graph-based network reconstruction, regarding the rooms as primal space, and relationships between them as dual space, for instance applied on 2D building plans by Yang and Worboys (2015), or 3D building models by Boguslawski et al. (2011). Others generate routes in an indoor environment based on centrelines of polygons in building plans (Meijers et al., 2005). These lines represent the skeleton of the polygon, also called the Medial Axis (Blum, 1967).

\subsection{Door detection}

A main requirement for the construction of an indoor navigation graph is an awareness of the locations of doors and traversable openings in walls, and which two indoor spaces they connect. There are various methods of doorway detection in indoor point clouds discussed in research. Díaz-Vilariño et al. (2014) and Díaz-Vilariño et al. (2015) use Generalized Hough Transforms (GHT) (Ballard, 1981) to detect edges in point clouds, in this way detecting door-shapes. Díaz-Vilariño et al. (2016) determine wall planes in the point cloud, which are then read as a binary image, with pixel values dependent on whether they contain points. These methods are however very dependent on a predetermined size and shape of the door, and are also prone to detect false positives.

In order to prevent this, one can make use of the trajectory of the mobile laser scanner, for instance by detecting doors that are walked trough. An example of this is described by both DíazVilariño et al. (2017) and Staats et al. (2018) who analyse the height of the original point cloud above the trajectory. Wherever a drop in height is observed, it is assumed the trajectory is passing a door frame. This method will only work in buildings with doors that are not of the same height as the ceiling. Another method that makes use of the trajectory of the scanner is described by Nikoohemat et al. (2017) and Nikoohemat et al. (2018), who apply ray-casting from the trajectory to detect openings in walls. An opening is defined as a door when the trajectory is passing close by the door centre.

\section{THEORETICAL BACKGROUND}

\subsection{IndoorGML network structure}

IndoorGML OGC (2014) is a standard developed by the Open Geospatial Consortium (OGC), especially focused on modelling the interior of buildings for navigation purposes. This includes the relationships between different indoor spaces in the form of a Node-Relation Graph (NRG), which is based on the idea of duality. In the NRG indoor spaces (e.g. rooms and corridors) are the nodes, and the relationships between them are described by edges. There are three different types of NRGs: Adjacency, Connectivity and Accessibility. If two indoor spaces are beside each other (touching), there is an edge connecting them in the Adjacency NRG. If there is also a door in the wall they share, it means there is an edge connecting them in the Connectivity NRG. In the Accessibility NRG more specific information is saved, such as whether a certain type of user (e.g. wheelchair user) can traverse a node or edge (e.g. representing stairs).

There are two ways in which a NRG can be represented. First of all there is the thin wall model, in which indoor spaces have a node, and their connections are shown with edges. Secondly there is the thick wall model, in which also walls and doors are represented by nodes. The relationships between walls, doors and indoor spaces are shown as edges. Including walls and doors in the network improves knowledge on the indoor environment, which is advantageous for navigation. In case of a connectivity graph the walls can be left out of the model, because they cannot be traversed, but doors are assigned a node. An example of a thick wall connectivity graph for a floor plan is shown in Figure 1 .

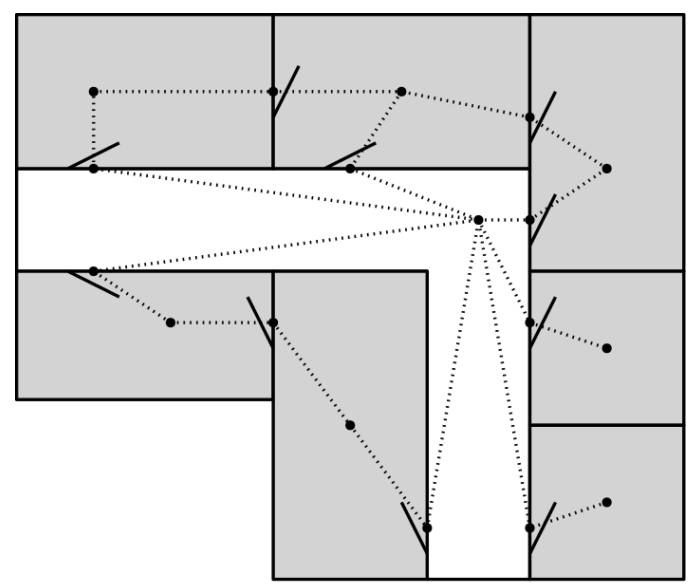

Figure 1. An example of a thick wall Connectivity NRG (dotted line) for a floor plan

For navigation purposes the Connectivity and Accessibility NRGs are most useful to consider, making them the main objectives of this research, which is to automatically extract a navigation graph with IndoorGML structure from a point cloud.

\subsection{Voxelisation and walkable space}

3.2.1 Voxelisation: Voxelisation of a point cloud can be applied in order to structure it, and so that it can be processed faster and more easily later. Voxels, also described as "volumetric pixels" (Nourian et al., 2016), can be used as a 3D grid structure on the point cloud, having cells of a fixed $\mathrm{x}, \mathrm{y}$ and $\mathrm{z}$ dimension. For each cell it is determined whether it is filled with point cloud 
points, or empty. It is applied by Broersen et al. (2015) by using an octree structure, in which the bounding box of the point cloud is recursively divided into eight octants, until the smallest scale is achieved. The smallest level octants are used as voxels in the methods of this paper.

3.2.2 Walkable voxel detection: Staats et al. (2018) developed a method to classify a voxelised indoor point cloud into horizontal surfaces, stairs, slopes and other types of elements. This is done by voxelising the point cloud and the mobile laser scanner trajectory. By analysing the height differences in the trajectory stairs, slopes and horizontal parts are classified. By projecting the classified trajectory voxels down to the voxelised point cloud this classification is passed through, and these voxels are used as seed points in a region growing algorithm, to include more voxels to the floor, stair and slope elements. The neighbour searching in the region growing algorithm takes into account an 8-neighbourhood of the voxel in the horizontal plane (see Figure 2).

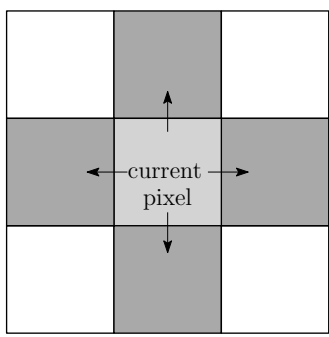

4-connectivity

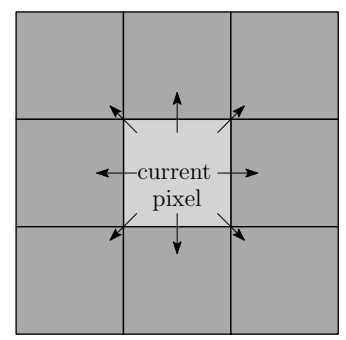

8-connectivity
Figure 2. 4- versus 8-neighbourhood of a pixel

After this doors are identified by looking at height differences in the voxelised original point cloud above the trajectory. This algorithm also checks the distance to the sides of the doors. The door detection process was not automated yet, which limits the automatic detection of different spaces. After the voxelised point cloud is region grown into floor regions, the walkable space is identified by subtracting furniture elements.

\subsection{D MAT}

As discussed in Section 2, 2D Medial Axis has already been used in the generation of navigation networks from 2D floor plans. In this research the 3D MAT for geographical point clouds (see Peters (2018)) is computed from an indoor point cloud and used for wall detection. The 3D MAT method of Peters consists of two steps. First, the Unstructured 3D MAT, in which all medial balls are calculated for the point cloud, using a ball-shrinking algorithm (Ma et al., 2012). Second, the Structured 3D MAT, in which the medial balls are grouped into medial sheets, analogous to branches of the 2D MAT, by region growing.

3.3.1 Unstructured 3D MAT: The 3D MAT consists of medial balls. A medial ball with centre $(c)$ and radius $(r)$ has the following three characteristics: 1) the surface of a medial ball touches two or more points of the point cloud (points $p$ and $q$ ), 2 ) the normal vectors of points $p$ and $q$ point towards centre $c$, meaning that the ball surface is tangent to the surface at $p$ and $q, 3)$ a medial ball does not contain any points of the original point cloud. An example of the MAT for a 2D shape is shown in Figure 3. In order to obtain medial balls in a point cloud, the ball-shrinking algorithm of Ma et al. (2012) is used.

3.3.2 Structured 3D MAT: The set of medial balls can be structured by grouping them into sheets, using a region growing

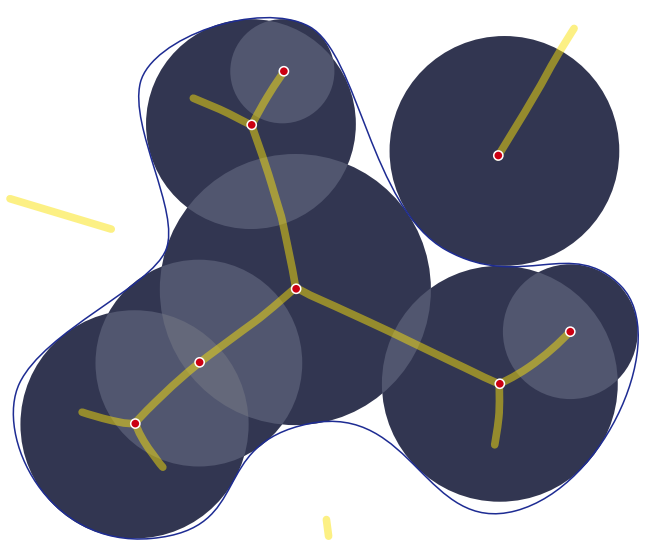

Figure 3. Some medial balls of a shape and in yellow the corresponding medial axis (Peters, 2018)

algorithm. A medial ball is selected as seed point, and its neighbours are added to the same medial sheet if they overlap. Moreover, the separation angle $\theta$ of a medial ball, which is the angle between vector $\overrightarrow{c p}$ and $\overrightarrow{c q}$ (see Figure 4) is taken into account. $\theta$ of the neighbour should not differ more than a threshold value from $\theta$ of the seed point, to ensure similarity. As a result of this multiple medial sheets are computed for the point cloud, which can be used to analyse structural elements in the point cloud.

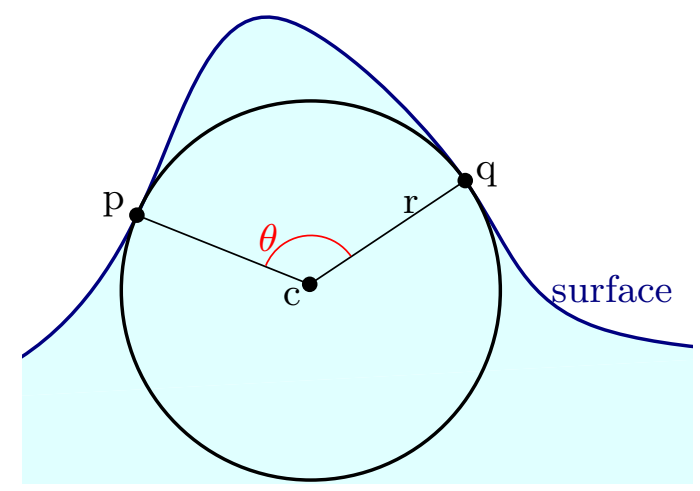

Figure 4. Key geometric characteristics of a medial ball

\section{METHODOLOGY}

The methodology of this research is based on the theories discussed in Section 3. First of all, 3D MAT is applied on an indoor point cloud, and MAT sheets formed inside walls are retained, as described in Section 4.1. The MAT sheets, original point cloud and trajectory are then voxelised. Using these MAT sheets the location of doors are detected, and original point cloud voxels on the floor inside the door frame are removed (see Section 4.2). This ensures that the walkable voxel space, as generated by the method of Staats et al. (2018), is classified into different indoor spaces. With these results a navigation graph in the manner of an IndoorGML Connectivity NRG is created (Section 4.3). A summary of all steps is shown in Figure 5.

\subsection{Wall detection using 3D MAT}

When the 3D MAT algorithm is applied on an indoor point cloud, the resulting medial sheets are amongst others formed between furniture objects, in hallways between walls, but also in the empty space inside walls that were scanned on both sides (see Figure 


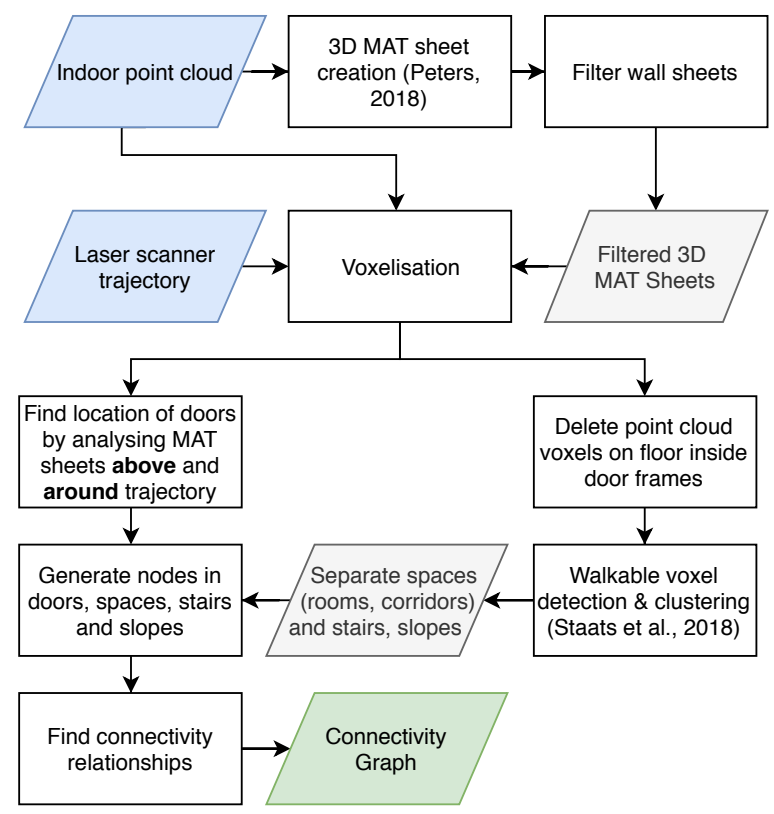

Figure 5. Overview of the steps in the methodology

6). These last type of sheets can be retained by applying filter parameters, based on the geometric characteristics of medial balls belonging to those sheets, as shown in Figure 4. Medial balls that are formed inside walls have two distinct characteristics, namely: 1) the radius $r$ is half the thickness of a wall, which is relatively small, and 2) the separation angle $\theta$ is approaching $180^{\circ}$. To illustrate this, in Figure 7 these characteristics of a ball inside a wall are shown.
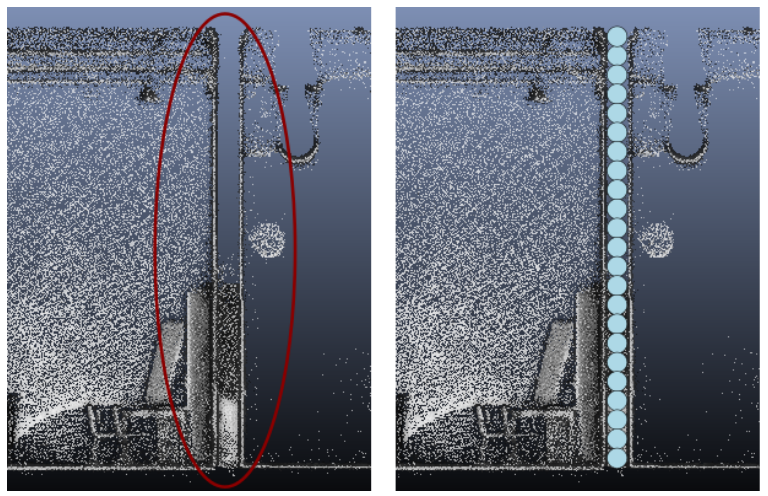

Figure 6. Left: empty space in wall scanned on both sides, right: some medial balls formed inside the wall

Moreover, the normal vectors of the centre points belonging to the medial balls in a wall sheet will point sideways, because a wall is perpendicular to the horizontal, meaning that the $\mathrm{z}$-component of normal vectors will be close to 0 . On top of this all, walls are generally large structures in a building, so the sheets inside them will have a large total number of points, which is also used as a filter parameter. In order to filter sheets based on the radius and separation angle, the median of each parameter in a sheet is taken to get a sample value of all points. For the z-component of the normal vectors in a sheet first the absolute value is calculated, after which also the median value of all points is calculated. A summary of all filter parameters is shown in Table 1. Results after testing different values for the parameters are discussed in Section 5.1.

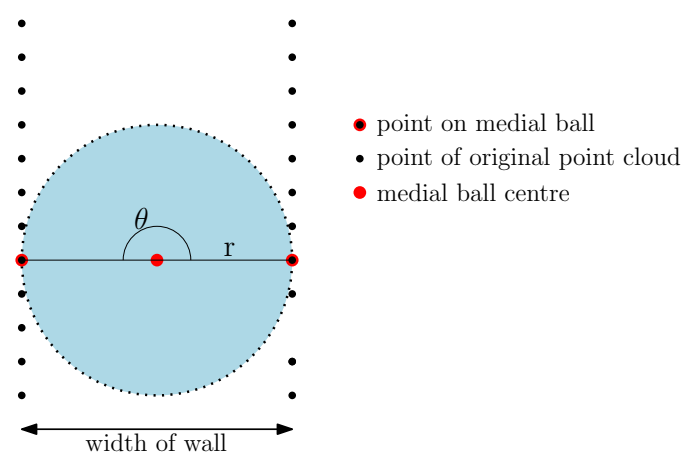

Figure 7. Local geometry of a medial ball (blue) inside a wall (side view)

\begin{tabular}{|l|l|}
\hline Parameter & Value in MAT sheet \\
\hline Separation angle $\theta$ & Median approaching $180^{\circ}$ \\
\hline Radius $r$ & Median $<$ half max. thickness walls \\
\hline Z-component normal $n_{z}$ & Absolute median approaching 0 \\
\hline Total number of points & High \\
\hline
\end{tabular}

Table 1. Theoretical parameters for filtering MAT sheets

\subsection{Doorway detection}

After filtering the MAT sheets to retain wall sheets, the results can be used for doorway detection. In order to do this, the wall sheets, original point cloud and laser scanner trajectory are all voxelised with the same voxel size and origin parameters. In this method not only the location of the door needs to be detected, but also the voxels in the original point cloud that lie on the floor inside a door frame (see Figure 8). The goal of this is to create a gap between two separate indoor spaces, which will ensure separate floor regions being created in the region growing step of walkable voxel detection (Section 3.2).
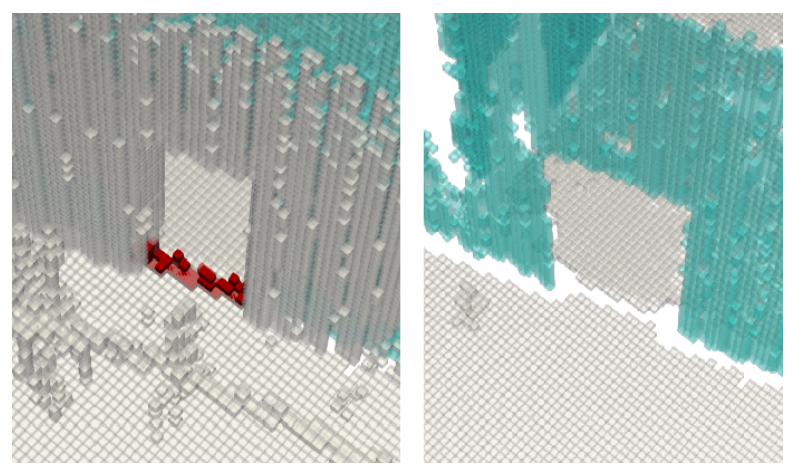

Figure 8. Left: Voxels (in red) of the original point cloud that lie on the floor inside a doorway, right: voxels inside door frame removed, creating a gap

There are two situations for the door detection. The first situation, shown in Figure 8, occurs when there is a wall above the door. For each trajectory voxel it is checked whether there exists a wall sheet voxel above it. If so, the trajectory voxel is marked as the location of a door.

In order to create the gap between two rooms inside the door frame, so that the rooms are separated in region growing, it is also checked for all original point cloud voxels whether they lie below a wall sheet voxel.

In the second situation considers doors that are of the same height as the ceiling, which means that there is no wall between the top 
of the door frame and the ceiling, so the first situation cannot be used. In order to detect the location of doors, and which voxels lie on the floor inside doors, the MAT sheet voxels around the trajectory are inspected, to check whether there is a wall on both sides of the trajectory (compare Figure 9 and 10).

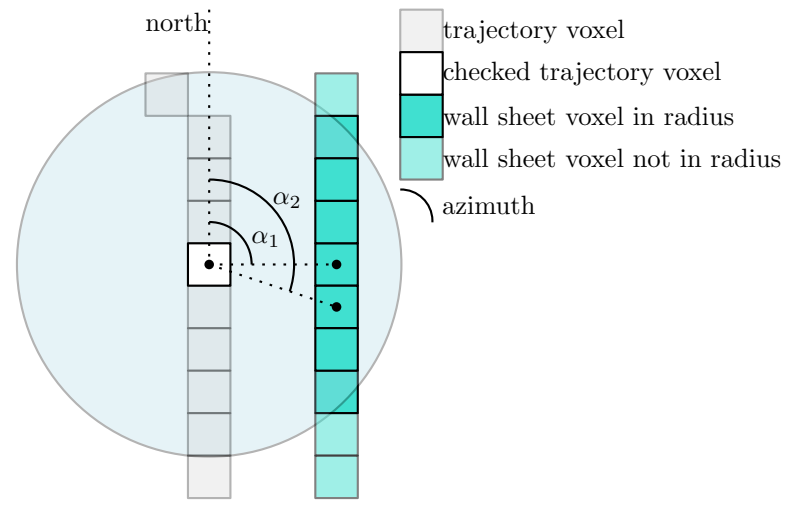

Figure 9. Top view of situation when trajectory goes along a wall

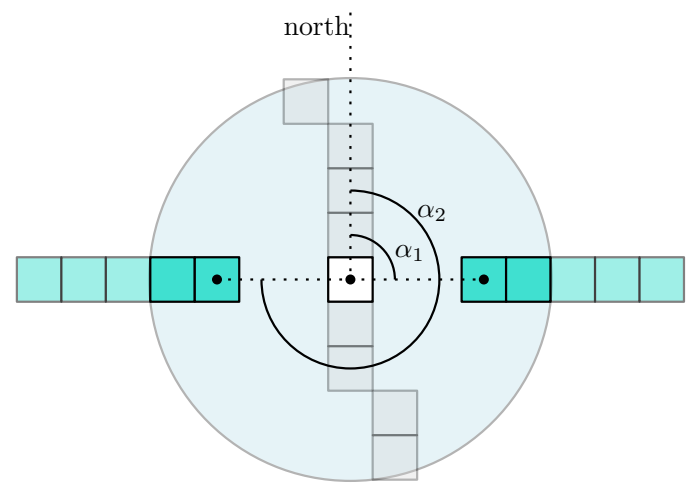

Figure 10. Top view of situation when trajectory passes a doorframe (legend in Fig. 9)

For each trajectory voxel it is checked which MAT sheet voxels fall inside a certain radius around it. For all of these voxels, the azimuth, $\alpha$, is calculated (see Figure 9). Then, they are all paired with each other, meaning that for a trajectory voxel with $n$ MAT sheet voxels in its radius, there will be $\left(\begin{array}{l}n \\ k\end{array}\right)=\left(\begin{array}{c}n ! \\ k !(n-k) !\end{array}\right)$ pairs. For all pairs the absolute difference in azimuth is calculated, as $\left|\alpha_{1}-\alpha_{2}\right|$. When, for one or more pairs, this value $\approx 180^{\circ}$, the trajectory voxel is selected as door location. This does not occur when a trajectory goes alongside a wall (see Figure 9), so there is no pair of voxels inside the radius where $\left|\alpha_{1}-\alpha_{2}\right| \approx 180^{\circ}$.

In order to get all voxels on the floor inside the doorframe, the pair of MAT sheet voxels that fulfils the condition $\left|\alpha_{1}-\alpha_{2}\right| \approx 180^{\circ}$ is selected. A virtual line is drawn between the pair, and a line voxelisation algorithm is applied to get a sequence of voxels on this line at the height of the trajectory, see Figure 11. All voxels of the original point cloud that lie below this line are not taken into account for the region growing of walkable voxels.

In a narrow corridor it can happen that many consecutive doors are detected falsely by the method looking at MAT sheet voxels, because the wall is close on both sides of the trajectory. This is prevented by analysing the number of consecutive trajectory voxels that are detected as a door, and removing them as door nodes when this sequence is longer than the maximum thickness of a wall in voxels.
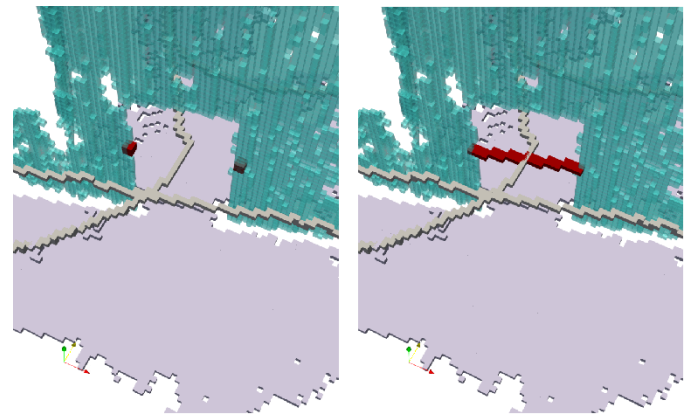

Figure 11. Left: Pair of MAT sheet voxels inside doorframe (red), right: voxel line drawn between pair of voxels

\subsection{Connectivity graph creation}

In order to create a connectivity graph in IndoorGML NodeRelation Graph (NRG) manner, nodes have to be placed in spaces, doors, slopes and stairs, and connectivity relationships between them have to be determined.

4.3.1 Node placement: The locations of doors are known from the method described in Section 4.2, in which also the voxels inside the door frame are removed. Because of this, together with the region growing in which floors, stairs and slopes are classified, the floor is segmented into different indoor spaces. From these separate spaces nodes can be extracted by selecting a point inside the space. This can be done by taking the mean $\mathrm{x}, \mathrm{y}, \mathrm{z}$ for a convex shaped room, or by selecting a random floor voxel as a node when the room is concave.

As no specific way is discussed in the IndoorGML standard on the placement of nodes in stairs and slopes, it has been decided to place a node at the top and bottom of a stairway, in order for it to connect well to the rest of the graph. For this the beginning and the end of a stair or slope are detected, by looking at the places in the trajectory when the type, as described in Section 3.2, changes from floor to slope or stair.

4.3.2 Connectivity determination: In order to find which nodes are connected the door, stair and slope nodes are regarded. It is assumed that all separate indoor spaces are connected to other spaces by either doors, stairways or slopes. A door always lies between two spaces; two floors, a floor and a stair or slope, or two stairs or slopes. These spaces are detected by finding the closest spaces to the door node. For stair and slope nodes: the bottom of a stairway or slope is always connected to either a space or a door, and the top of the stair or slope. For the top node this is applied vice versa. The network is created by connecting the stair and door points to the points in the spaces.

\section{RESULTS AND DISCUSSION}

The methods are applied on a point cloud gathered in a part of a university faculty. It includes a hallway, with some rooms and a staircase leading to another room adjacent to it, and a large hall with a large staircase in the middle incorporating multiple floors. It was gathered with a ZEB-REVO laser scanner. The results of the methods applied to get a connectivity graph for this point cloud are discussed in this chapter.

\subsection{Wall detection by 3D MAT}

The 3D MAT sheet parameters, that were defined in Section 4.1, have been tested on the point cloud to find the most optimal values. First of all different values of the separation angle were tried, 
and with keeping only sheets with a median of $>0.8 \pi \mathrm{rad}$ no wall sheets were filtered out, and no unnecessary sheets were kept. The same approach was used for the radius, which gave the best value of keeping all sheets with a median of $<0.4 \mathrm{~m}$, and for the z-component of the normal vector with a value of $<0.2$ only wall sheets were kept. When a sheet had less than 1000 points, it was never generated inside wall. A summary of the results is shown in Table 2 .

\begin{tabular}{|l|l|}
\hline Parameter & \multicolumn{1}{|c|}{ Value in MAT sheet } \\
\hline Separation angle $\theta$ & Median $>0.8 \pi \mathrm{rad}$ \\
\hline Radius $r$ & Median $<0.4 \mathrm{~m}$ \\
\hline Z-component normal $n_{z}$ & Absolute median $<0.2$ \\
\hline Total number of points & $>1000$ \\
\hline
\end{tabular}

Table 2. Theoretical parameters for filtering MAT sheets

\subsection{Doorway detection}

In the door detection method proposed in this thesis only doors that are walked through can be detected. There are 13 doors in total, of which 7 are walked through. Most of the 6 doors that were not walked through belong to rooms that were passed by, but not visited. In the point cloud used, 7 doors are walked through, of which all locations are detected. There are no doors detected at locations where there is no door, so no false positives. For one of the 7 doors that is detected, the indoor space is not split into two separate spaces. The reason for this is shown in Figure 12. This is caused by the fact that in the step of region growing of floors the 8-neighbourhood of a voxel is taken into account, so voxels that touch diagonally are counted as neighbours.
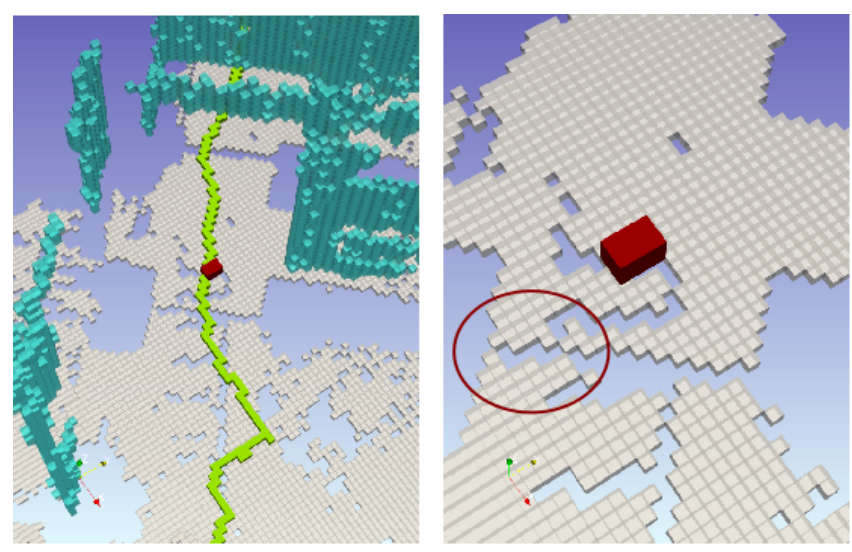

Figure 12. Door location is detected (left), but floor not separated into two indoor spaces, because touching floor voxels (right)

\subsection{Connectivity graph creation}

After applying the walkable space detection step, with voxels inside door frames taken out of region growing, the results in Figure 13 are obtained. Different colours indicate separate spaces. All spaces that were visited by the ZEB-REVO laser scanner have been automatically assigned a separate identifier. The dark blue floor patch is the corridor mentioned, and the yellow floor patch the large hall. The orange patch is the second floor from the corridor.

The connectivity network resulting from the node allocation and connectivity determination steps is shown in Figure 14. All rooms and doors have been assigned one node (orange and green

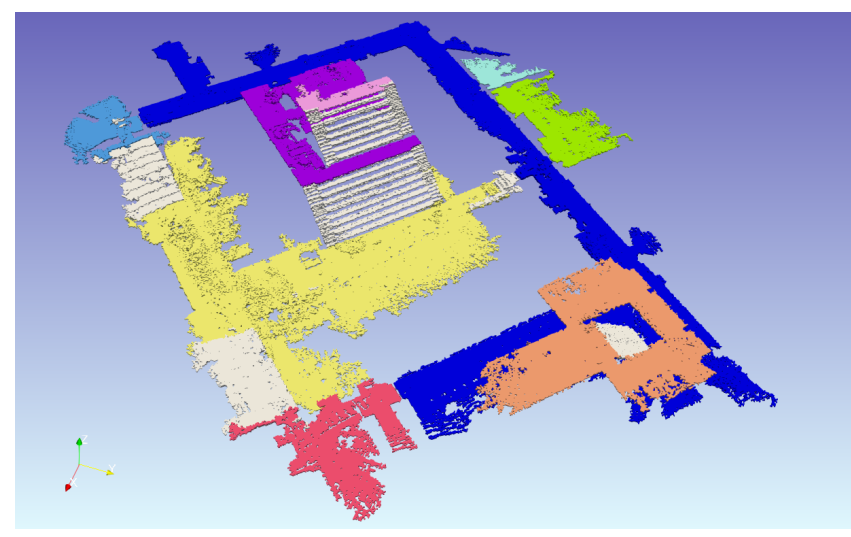

Figure 13. Resulting separate indoor spaces indicated with different colours, stairs and slopes shown in white

respectively), and stairs and slopes are indicated with a red node at their top and bottom. Connectivity relationships are shown by the black lines. As can be seen, large spaces with multiple connections (such as the corridor and large hall) also have only one node. This makes routes that go through these spaces not always representative.

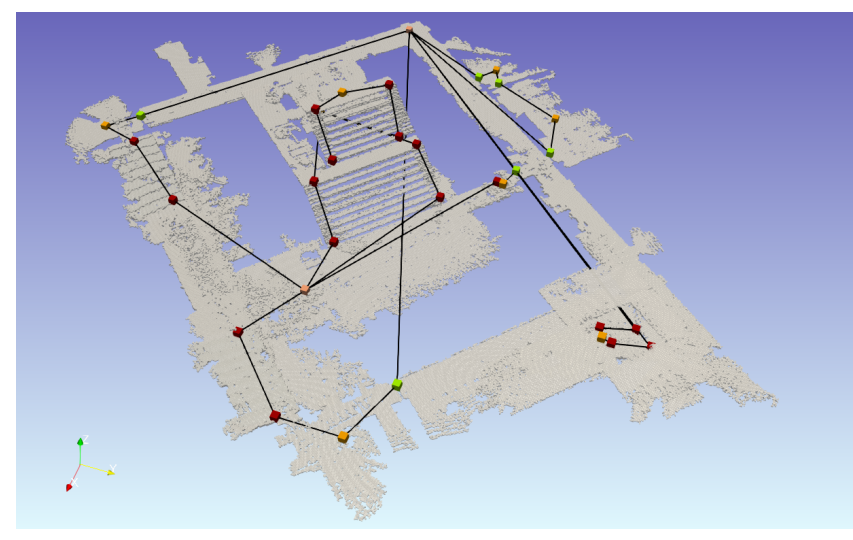

Figure 14. Connectivity network automatically generated from walkable space $($ red $=$ stair or slope, green $=$ door, orange $=$ room)

\subsection{Discussion}

The first results produced by the method proposed are promising. A connectivity graph is generated between indoor spaces, and doors, stairs and slopes are included. There are some points of discussion that came forth while implementing the methods.

First of all the filtering parameters of the sheets could be dependent on the type of indoor environment scanned, and especially the total number of points in a MAT sheet is very dependent on the point cloud density. Secondly only walls that were scanned on both sides are detected, because medial balls can only be created inside those walls. However, walls that were not scanned on both sides are not needed to detect doors, because when a wall has been scanned on only one side, it automatically means it has not been walked through. Lastly it can be noted that the output connectivity graph is not very suitable for navigation purposes yet. Because each indoor space has only one node, especially routes in corridors and large spaces are not representative of real paths. 


\section{CONCLUSIONS AND FUTURE WORK}

In this paper an automatic method is proposed to obtain a navigation graph with IndoorGML Connectivity NRG structure from a point cloud and laser scanner trajectory, by applying the 3D MAT algorithm, and filtering it to obtain MAT sheets formed inside walls. These wall sheets are then used to detect the location of doors in the voxelised point cloud, and also the voxels on the floor inside the door frame. The latter are then removed from the region growing step, in which walkable voxels are identified and classified into floor, stair and slope voxels (Staats et al., 2018). This results in regions being grown as separate indoor spaces, which are then used, together with the location of doors, stairs and slopes, to create nodes at those locations. Connectivity relationships are deducted, which can form the basis of an IndoorGML Connectivity NRG.

From the results shown in Section 5 the following conclusions can be drawn:

- The 3D MAT, as implemented by Peters (2018), can be applied to indoor point clouds, and filtered in order to retain only MAT sheets that were formed inside walls that were scanned on both sides.

- Door detection using the 3D MAT wall sheets show promising results. Only doors that are walked through are detected. Of 7 doors, the location of all could be detected. For one door the gap created in the door frame between two spaces was not adequate, causing them to be joined in the region growing of walkable voxels.

- From the location of doors, rooms, stairs and slopes a graph with IndoorGML Connectivity NRG structure can be created.

- This connectivity network is not directly suitable for shortest path calculations. When a large corridor has only one node, the routes leading through this node are not representative of a real path.

- Accessibility information such as the location of stairs can be directly retrieved from the connectivity graph. The width and height of doors could be obtained from the voxelised point cloud as well, by analysing height and width of voxels around the locations marked as doors. Other types of accessibility information, such as accessibility rights for certain persons, cannot be retrieved from an indoor point cloud.

- The methods presented for door detection require the dynamic laser scanner to pass through all doors in order for them to be detected. This can be seen as a weakness of the method, but also minimises the occurrence of false positives (doors being detected which are not really there) at the location of furniture that look like doors (closets), or doors that are permanently closed (see Figure 15).

These conclusions and other observations lead to the following future work:

- In order to ensure 8-separation in voxels that lie on the floor inside door frames a buffer could be created around the wall sheets voxels, and all voxels below this buffer could be taken out of the region growing step as well. This will ensure the gap between two spaces to be wide enough.

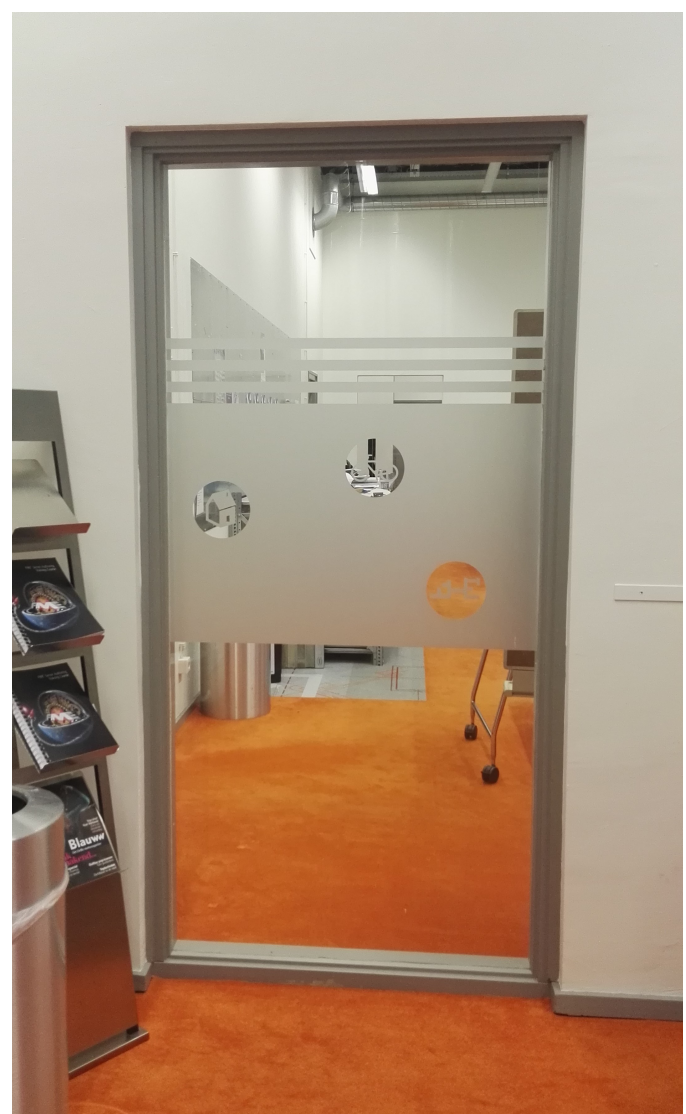

Figure 15. False door with a glass pane

- To make routes in a hallway more representative subspacing should be applied on some indoor spaces. This is a concept also described in IndoorGML. The authors of this paper are currently working on it, and have achieved promising results in long corridors, while more work is to be done on wide open spaces.

- The deducted connectivity relationships in this paper can be used as an input for a IndoorGML Connectivity and Accessibility NRG, which is something that the authors are currently working on as well.

\section{References}

Alattas, A., Zlatanova, S., Oosterom, P. V., Chatzinikolaou, E., Lemmen, C. and Li, K.-J., 2017. Supporting indoor navigation using access rights to spaces based on combined use of IndoorGML and LADM models. ISPRS International Journal of Geo-Information 6(12), pp. 384

Ballard, D., 1981. Generalizing the Hough transform to detect arbitrary shapes. Pattern recognition 13(2), pp. 111-122.

Blum, H., 1967. A transformation for extracting new descriptors of shape. Models for Perception of Speech and Visual Forms pp. 362-380.

Boguslawski, P., Gold, C. M. and Ledoux, H., 2011. Modelling and analysing $3 \mathrm{~d}$ buildings with a primal/dual data structure. ISPRS Journal of Photogrammetry and Remote Sensing 66(2), pp. 188-197.

Boguslawski, P., Mahdjoubi, L., Zverovich, V. and Fadli, F., 2016. Automated construction of variable density navigable networks in a $3 \mathrm{~d}$ indoor environment for emergency response. Automation in Construction 72, pp. 115-128. 
Bosché, F., Guillemet, A., Turkan, Y., Haas, C. T. and Haas, R., 2014. Tracking the built status of MEP works: Assessing the value of a scan-vs-BIM system. Journal of Computing in Civil Engineering 28(4), pp. 05014004.

Broersen, T., Fichtner, F., Heeres, E., De LIefde, I., Rodenberg, O., Meijers, B., Verbree, E., Van der Spek, S. and Ten Napel, D., 2015. Project pointless: Identifying, visualising and pathfinding through empty space in interior point clouds using an octree approach. Geomatics Synthesis Project 2015/16.

Díaz-Vilariño, L., Boguslawski, P., Khoshelham, K., Lorenzo, H. and Mahdjoubi, L., 2016. Indoor Navigation From Point Clouds: 3D Modelling And Obstacle Detection. ISPRS - International Archives of the Photogrammetry, Remote Sensing and Spatial Information Sciences XLI-B4, pp. 275-281.

Díaz-Vilariño, L., Khoshelham, K., Martnez-Snchez, J. and Arias, P., 2015. 3d modeling of building indoor spaces and closed doors from imagery and point clouds. Sensors 15(2), pp. 3491-3512.

Díaz-Vilariño, L., Martínez-Sánchez, J., Lagela, S., Armesto, J. and Khoshelham, K., 2014. Door recognition in cluttered building interiors using imagery and lidar data. ISPRS - International Archives of the Photogrammetry, Remote Sensing and Spatial Information Sciences XL-5, pp. 203-209.

Díaz-Vilariño, L., Verbree, E., Zlatanova, S. and Diakité, A., 2017. Indoor Modelling From SLAM-Based Laser Scanner: Door Detection To Envelope Reconstruction. ISPRS - International Archives of the Photogrammetry, Remote Sensing and Spatial Information Sciences XLII-2/W7, pp. 345-352.

Fichtner, F. W., Diakité, A. A., Zlatanova, S. and Voûte, R., 2018. Semantic enrichment of octree structured point clouds for multi-story 3d pathfinding. Transactions in GIS 22(1), pp. $233-248$.

Ge, S. and Cui, Y., 2002. Dynamic motion planning for mobile robots using potential field method. Autonomous Robots 13(3), pp. 207-222.

Hong, S., Jung, J., Kim, S., Cho, H., Lee, J. and Heo, J., 2015. Semi-automated approach to indoor mapping for $3 \mathrm{~d}$ as-built building information modeling. Computers, Environment and Urban Systems 51, pp. 34-46.

Koren, Y. and Borenstein, J., 1991. Potential field methods and their inherent limitations for mobile robot navigation. In: Proceedings. 1991 IEEE International Conference on Robotics and Automation, IEEE Comput. Soc. Press.

Ma, J., Bae, S. W. and Choi, S., 2012. 3d medial axis point approximation using nearest neighbors and the normal field. The Visual Computer 28(1), pp. 7-19.

Meijers, M., Zlatanova, S. and Pfeifer, N., 2005. 3d geoinformation indoors: structuring for evacuation. In: Proceedings of Next generation $3 D$ city models, Vol. 6, Germany Bonn.

Mirza, R., Tehseen, A. and Kumar, A. V. J., 2012. An indoor navigation approach to aid the physically disabled people. In: 2012 International Conference on Computing, Electronics and Electrical Technologies (ICCEET), IEEE.

Nakagawa, M. and Nozaki, R., 2018. Geometrical Network Model Generation Using Point Cloud Data for Indoor Navigation. ISPRS Annals of Photogrammetry, Remote Sensing and Spatial Information Sciences IV-4, pp. 141-146.
Nikoohemat, S., Peter, M., Elberink, S. O. and Vosselman, G., 2017. Exploiting Indoor Mobile Laser Scanner Trajectories for Semantic Interpretation of Point Clouds. ISPRS Annals of Photogrammetry, Remote Sensing and Spatial Information Sciences IV-2/W4, pp. 355-362.

Nikoohemat, S., Peter, M., Elberink, S. O. and Vosselman, G., 2018. Semantic interpretation of mobile laser scanner point clouds in indoor scenes using trajectories. Remote Sensing 10(11), pp. 1754

Nourian, P., Gonçalves, R., Zlatanova, S., Ohori, K. A. and Vo, A. V., 2016. Voxelization algorithms for geospatial applications. MethodsX 3, pp. 69-86.

OGC, 2014. OGC Indoor GML.

Peters, R., 2018. Geographical point cloud modelling with the 3D medial axis transform. PhD thesis, TU Delft.

Sithole, G., 2018. Indoor Space Routing Graphs: Visibility, Encoding, Encryption and Attenuation. ISPRS - International Archives of the Photogrammetry, Remote Sensing and Spatial Information Sciences XLII-4, pp. 579-585.

Staats, B. R., Diakité, A. A., Voûte, R. L. and Zlatanova, S., 2017. Automatic Generation of Indoor Navigable Space Using a Point Cloud and its Scanner Trajectory. ISPRS Annals of Photogrammetry, Remote Sensing and Spatial Information Sciences IV-2/W4, pp. 393-400.

Staats, B. R., Diakité, A. A., Voûte, R. L. and Zlatanova, S., 2018. Detection of doors in a voxel model, derived from a point cloud and its scanner trajectory, to improve the segmentation of the walkable space. International Journal of Urban Sciences pp. 1-22.

Volk, R., Stengel, J. and Schultmann, F., 2014. Building information modeling (BIM) for existing buildings - literature review and future needs. Automation in Construction 38, pp. 109-127.

Yang, L. and Worboys, M., 2015. Generation of navigation graphs for indoor space. International Journal of Geographical Information Science 29(10), pp. 1737-1756. 